\title{
Application of elements of quantum mechanics in analysing AC conductivity and determining the dimensions of water nanodrops in the composite of cellulose and mineral oil
}

\author{
P. Żukowski • K. Kierczyński (D) T. N. Kołtunowicz • P. Rogalski • \\ J. Subocz
}

Received: 26 October 2018/Accepted: 14 January 2019/Published online: 11 February 2019

(C) The Author(s) 2019

\begin{abstract}
The paper presents a model of hopping conductivity at direct and alternating current, developed on the basis of the quantum phenomenon of electron tunnelling between neighbouring potential wells. The tunnelling takes place between neutral potential wells, resulting in an electrical dipole and additional thermally activated polarisation. An important parameter of the model is the time of existence of the dipole (relaxation time). After this time, the electron with the probability $p$ can hop to the third well, which causes a direct current flow, or return with the probability $(1-p)$ to the first well, thus causing the flow of high frequency current. The model shows that for direct or low frequency current, the current density and conductivity do not depend on the frequency. In the high frequency region, the current density does not depend on the frequency either. Lowfrequency conductivity is $2 p$ times smaller than highfrequency conductivity. In the transitional region there
\end{abstract}

P. Żukowski · K. Kierczyński (ه) ·

T. N. Kołtunowicz · P. Rogalski

Department of Electrical Devices and High Voltage

Technology, Faculty of Electrical Engineering and

Computer Science, Lublin University of Technology, 38a,

Nadbystrzycka Street, 20-618 Lublin, Poland

e-mail: k.kierczynski@pollub.pl

J. Subocz

Department of Electrotechnology and Diagnostics, Faculty of Electrical Engineering, West Pomeranian University of Technology, 37, Sikorskiego Street, 70-313 Szczecin, Poland is a frequency dependence of the current density. A formula was derived for the dependence of the relaxation time on the distance between neighbouring potential wells and temperature. The dependence of the hopping probability on activation energy, potential dipole energy and temperature was determined. A model of hopping conductivity at direct and alternating current was used to analyse the experimental frequency dependence of conductivity and permittivity of the composite of electrotechnical pressboard, mineral oil and water nanoparticles. It was found that there is a high compatibility of the experimental course with the model. It was also found that in the composite of electrotechnical pressboard, mineral oil and water nanodrops there are at least two mechanisms of changes in low frequency and high frequency conductivity. For the low-frequency stage, the value of the probability of $p_{1} \approx 0.2$ and the relaxation time $\tau_{1} \approx 0.02 \mathrm{~s}$. The probability of hops for the highfrequency growth stage is $p_{2} \approx 0.0005$ and the value of relaxation time $\tau_{2} \approx 2 \times 10^{-7} \mathrm{~s}$. The occurrence of two episodes of increase in conductivity is related to the presence in the composite of electrotechnical pressboard, mineral oil and water nanoparticles of differences in distances between neighbouring nanoparticles. For a large number of nanoparticles randomly distributed in the percolation channel, the distribution of the probability of the occurrence of the distance is in a good approximation normal. This means that in the percolation channel there are pairs of neighbouring wells, the distances between which are 
both much smaller than the average and much larger. A pair of wells, the distance between which is smaller than medium, participates in the conductivity on the high-frequency stage with a short relaxation time. On the other hand, a pair of neighbouring wells for which the distance is greater than average causes a high resistance to DC or low-frequency current flow as well as a higher relaxation time. On the basis of the dependences designated in laboratory tests of lowfrequency conductivity $\left(10^{-4} \mathrm{~Hz}\right)$ from the moisture content, using the elements of quantum mechanics, the dimensions of water nanoparticles in electrotechnical pressboard impregnated with mineral insulating oil were determined. It was found that nanoparticles contain on average about 200 molecules of water, and their diameters are about $2.24 \mathrm{~nm}$. This approach allowed to calculate the dielectric permittivity of cellulose and to determine that water nanoparticles are located inside the cellulose fibres.

\section{Graphical abstract}

\section{Introduction}

For about a hundred years the production of highvoltage-power transformers has relied on insulation in the form of cellulose paper, impregnated by insulating oil. Paper-oil insulation, under long-term exploitation, undergoes aging phenomena, which are accompanied by cellulose depolymerisation processes, (Gilbert et al. 2010; Jalbert et al. 2015) take place, as a result of which the molecules of water precipitate directly in the cellulose fibers. However, the main source of moisture in the cellulose is moisture that penetrates into the transformer and dissolves in the transformer oil. The water is then supplied by cellulose oil and absorbed by it, this happens because the solubility of water in cellulose is more than a 1000 times higher than in transformer oil (Oommen 1983). These factors cause that during long-term operation the moisture content in the cellulose-mineral oil composite gradually increases from the initial level of about $0.8 \%$ by weight up to even more than $5 \%$ by weight. The

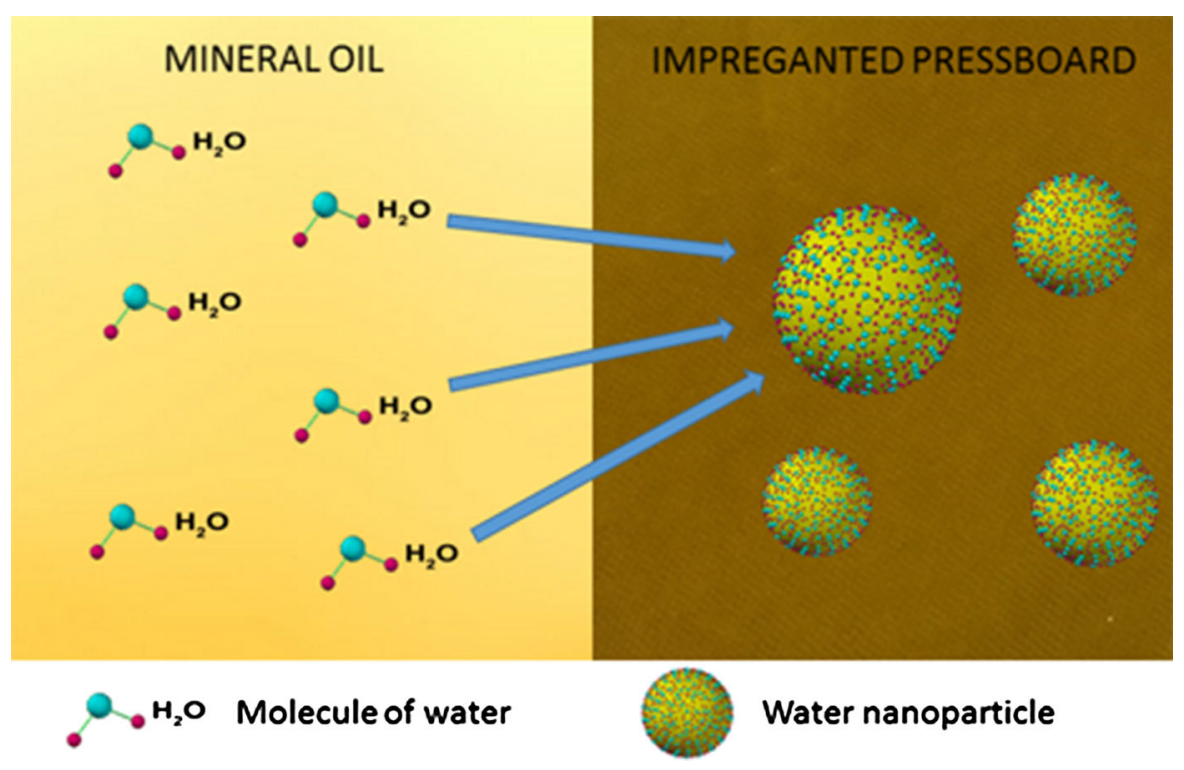

Keywords Electrotechnical pressboard - Mineral oil · Moisture content · Water nanoparticle · Quantum mechanics $\cdot$ AC conductivity growth of moisture content in paper-oil insulation significantly reduces its quality and the reliability work power transformers.

It follows that the level of moisture content in the fixed insulation of power transformers should be specified as accurately as possible. To determine the moisture content in paper-oil insulation in power 
transformers non-destructive electrical methods are used. These methods can be divided into: measurements in the Frequency Domain Spectroscopy (FDS) (Ekanayake et al. 2006; Jaya et al. 2013; Zhang et al. 2014; Walczak et al. 2006) and time domain measurements: Return Voltage Measurement (RVM) (Dey et al. 2010; Saha 2003) and Polarisation Depolarisation Current (PDC) (Wolny and Kędzia 2010; Saha and Purkait 2004).

The analysis of dielectric relaxation processes in the field of frequency and time is based on models, each of which has a more or less reliable physical explanation.

The DC conductivity of paper and oil insulation is traditionally considered within the framework of ionic conductance models described, among others, in Mott and Gurney (1950). This monograph discusses the basic models of ionic conductivity. In the Schottky model, ions are bound to the matrix, and their release takes place by means of a thermally activated dissociation. Conductivity in this case can be saved in the form:

$\sigma_{S}=\frac{e^{2} \cdot a^{2} \cdot v_{0}}{6 \cdot k \cdot T} N \exp \left(-\frac{W}{k T}\right)$,

where $N$-ion concentration; $W$-generalized activation energy; $v_{0}$-the probability of ion hops; $a$-the distance to which the ion hops.

In the Poole-Frenkel model, ions can be found in both vacant and internode positions. In this case, the pattern for conductivity looks as follows:

$\sigma_{P F}=\frac{e^{2} \cdot a^{2} \cdot v_{0}}{6 \cdot k \cdot T} \sqrt{N \cdot N_{I}} \exp \left(-\frac{W}{k T}\right)$,

where $N$-concentration of ions in vacancies, $N_{\Gamma}$ concentration of ions in internodes.

It appears from formulas $(1,2)$ that in the case of ionic conductivity there should be a linear dependence of conductivity on the content of moisture in the pressboard. Based on the analysis of experimental results (Zukowski et al. 2014), it was found that the dependence of direct current conductivity on the moisture content is much stronger than the linear one (exponential), and the DC conductivity takes place via the quantum phenomenon of electron tunneling between water molecules.

Until recently, the basis for the analysis of AC test results was the Debye dielectric relaxation model
(Jonscher and Andrzej 1983), which, as it turned out, has several features that do not have experimental confirmation. Therefore, in the area of pulsation values $\omega=2 \pi \mathrm{f}$ lower than the inverse relaxation time $\tau(\omega<1 / \tau)$, a quadratic dependence of conductivity on frequency takes place in this model. In moistened pressboard impregnated by isolation oil, this dependence is weaker than the square one (Walczak et al. 2006). Next disadvantage of the Debye model is the reduction permittivity in the square from the frequency. Measurements of permittivity of impregnated electrotechnical pressboard with a specific moisture content prove that these dependencies are weaker (Jonscher and Andrzej 1983). To justify the occurrence of weaker frequency dependencies of conductivity and dielectric permittivity than square ones, a number of empirical models have been developed (Cole and Cole 1941, 1942; Havriliak and Havriliak 1997).

The most important unfavorable conclusion resulting from the above models is the argument that when the frequency of the electric field tends to zero (to constant voltage), conductivity also tends to zero. This is contradictory to before determined measurement results, which in the ultra-low frequency range have a certain conductivity value, close to the DC conductivity.

In works (Zukowski et al. 2014, 2015a) based on laboratory tests observed that the DC conductivity in the composite of cellulose, mineral oil and water nanoparticles takes place by hopping (tunneling) of electrons between the potential wells produced by water nanodrops.

In order to analyze obtained on the basis of laboratory tests the AC current of the composite of cellulose, mineral oil and water nanoparticles, the following model of hopping conductivity at alternating and direct current will be used. This model was initially created to describe the conductivity in the semiconductor material of gallium arsenide, illuminated with high doses of high-energy hydrogen ions (Żukowski et al. 2007) and was then extended in a series of works for the case of metal-dielectric nanocomposites see for example (Kołtunowicz et al. 2013).

When developing the model of hopping conductivity for direct and alternating current the following assumptions were made: 
- In the material there are near-neutral potential wells, located in sufficient vicinity, in which electrons are located.

- A quantum phenomenon of electron tunneling occurs between the potential wells (Fig. 1). A number of patterns are known for the probability of tunneling (electron hopping) in the $P(T)$ unit of time (Mott et al. 1979), specifying $P(T)$, but their common feature is that:

$P(T)=P_{0} \exp \left(-\beta \alpha r-\frac{\Delta W}{k T}\right)$,

where $P_{0}=$ const. - numeric factor; $r$-average distance between potential wells; $\exp (-\beta \alpha r)$ - the fall rate of the square of the electron wave function localized in the three-dimensional potential well; $\beta$-a numeric factor, the value of which is $\beta \approx(1.75 \pm 0.05)$ (Shklovskii and Efros 1984); $\alpha=1 / R_{0} ; R_{0}$-the radius of the location of the wave function of the hopping electron (Bohr radius); $\Delta W-$ activation energy; $k$-Boltzmann constant; $T$ temperature.

- External constant or alternating electric field forcing current is weak and does not change the probability of $P(T)$ electrons hopping from one neutral potential well to another, but only leads to asymmetry of their hops, which can be taken into account with the Debye factor (Mott et al. 1979):

$\exp \left( \pm \frac{e r E}{k T}\right)$

where $e$ - the charge of an electron, $r$ - the distance between the potential well from which the electron tunnels and the well to which it hops (tunnels); $E$ intensity of the electric field; $k$-Boltzmann constant; $T$-temperature.

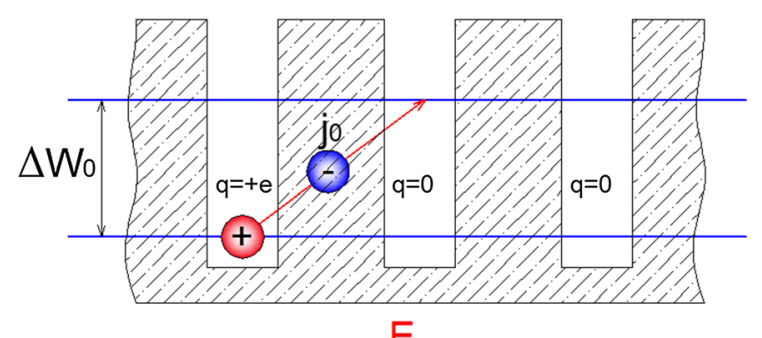

Fig. 1 The hop of the electron from one well to another in the opposite direction to the electric field

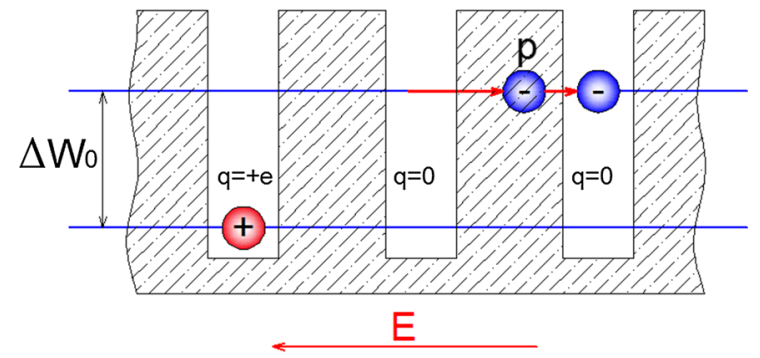

Fig. 2 The hop of the electron with the probability $p$ from the second to the third well

From formulas $(3,3)$ one can derive the formula for direct current conductivity for the case of tunneling between nearest neighbours (Ravich and Nemov 2002):

$\sigma(r, T=$ const $)=\sigma_{0} \exp \left(-\frac{\beta r}{R_{0}}-\frac{\Delta W}{k T}\right)$,

where $r$-average distance between potential wells; $R_{0}$ - the radius of the location of the wave function of the hopping electron (Bohr radius); $\beta$ - a numeric factor, the value of which is $\beta \approx(1.75 \pm 0.05)$ (Shklovskii and Efros1984); $\Delta W$-activation of conductivity, $k$-Boltzmann constant.

After the hop of the electron from one neutral well of potential to another, it stays in it for the life of the dipole (time of relaxation) $\tau$. At that time, the first well has a positive charge, while the second one has a negative charge. This means the occurrence in the material containing inert wells the potential of an additional thermally activated polarization caused by the hopping exchange of charges.

The hop itself generates the current of density $j_{0}$ (Fig. 1), described by a known formula (Żukowski et al. 2007):

$j_{0}=\sigma_{0} E_{0} \sin \varpi t$.

After the time $\tau$, two scenarios of further hops are possible. In the first variant after the dipole's existence time $\tau$ (relaxation time) the electron with the probability $p$ can hop in the direction opposite to the direction of the electric field to the third well (Fig. 2).

This causes the current to flow with a density determined by the formula (Źukowski et al. 2007):

$j_{+}=p \sigma_{0} E_{0} \sin \varpi(t-\tau)$.

In the second variant, the electron after the existence of the dipole $\tau$ with the probability of 
$1-p$ returns from the second well to the first well (Fig. 3), which leads to dipole failure and the flow of current with the density described by formula (Żukowski et al. 2007):

$j_{-}=-\sigma_{0} E_{0}(1-p) \sin \varpi(t-\tau)$.

The resultant current density $j$ will be described using the formula (Żukowski et al. 2007):

$$
\begin{aligned}
j= & j_{0}+j_{+}+j_{-}=\sigma_{0} E_{0}[\sin \varpi t+p \sin \varpi(t-\tau) \\
& -(1-p) \sin \varpi(t-\tau)] .
\end{aligned}
$$

The components $j_{+}$and $j_{-}$are phase shifted with respect to $j_{0}$, and therefore the resulting current density has both real and imaginary components. For a parallel equivalent circuit in which the AC conductivity is correctly measured, the actual current density component is ('̈ukowski et al. 2007):

$j_{r}=\sigma_{0} E_{0}(1-\cos \varpi t+2 p \cos \varpi \tau) \sin \varpi t$.

The analysis of formula (10) shows that for direct or low frequency current $(\omega \rightarrow 0)$, the current density does not depend on the frequency $\omega=2 \pi f$ and its value is:

$j_{r}=2 p \sigma_{0} E_{0} \sin \varpi t$.

In the high frequency area, the current density does not depend on the frequency $\omega$ :

$j_{r}=\sigma_{0} E_{0} \sin \varpi t$.

Formula (11) shows that direct and low-frequency conductivity in the case of hopping conductivity (tunneling) is:

$\sigma_{D C}=\sigma_{L}=2 p \sigma_{0}$.

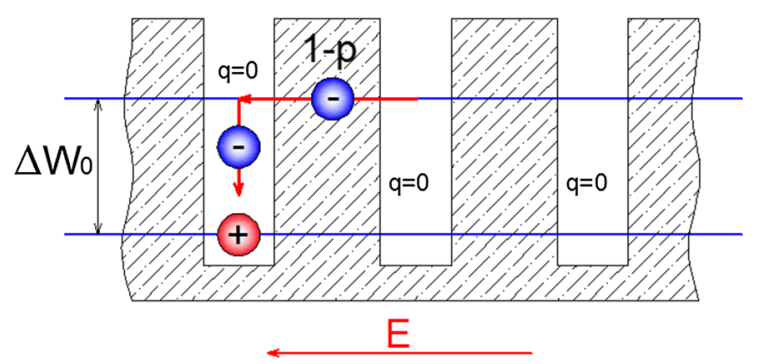

Fig. 3 The return of the electron with the probability of $1-p$ from the second well to the first well and dipole disappearance
On the other hand, in the area of high frequencies, see formula (12):

$\sigma_{H}=\sigma_{0}$.

In the transitional region for $\omega \tau \approx 1$ there is a frequency dependence of the current density which, following Mott et al. (1979), can be described with the formula

$j_{r} \sim f^{\alpha(f)}$,

where $\alpha(f)$-frequency coefficient.

As can be seen from further analysis, this coefficient is a function of frequency.

In Zukowski et al. (1997) it was shown that in systems of wells interacting with each other, time $\tau$ is not the same for all wells. Its value should depend on the distance between wells, which are distributed randomly in the material. The authors assumed that the distribution of $\tau$ times may be normal or similar to it.

For physical reasons, the probability distribution for the $\tau$ times should only be determined for the positive values of $\tau$, because the negative values of the $\tau$ times mean that the electron will return from the second well to the first well before it tunneled from the first well. The condition for determining the $\tau$ times only for positive values corresponds, among others, to Moyal's approximation of the Landau distribution (Nowak 2002). This distribution is described by the formula:

$F_{L(\tau)}=\frac{1}{\sigma_{m} \sqrt{1 \pi}} \exp \left(-\frac{\tau-\tau_{m}}{2 \sigma_{m}}-\frac{1}{2} \exp \left(-\frac{\tau-\tau_{m}}{\sigma_{m}}\right)\right)$,

where $\sigma_{\mathrm{m}}$-standard deviation; $\tau_{m}$ - expected value of the relaxation time.

Taking into account the Landau distribution of the probabilities of the occurrence of relaxation times $\tau$, formula (10) for the density of the actual AC component will be saved in the form:

$$
\begin{aligned}
j_{r}(\varpi)= & \sigma_{0} E_{0} \int_{0}^{\infty} F_{L}(\tau)(1-\cos \varpi t \\
& +2 p \cos \varpi \tau) \sin \varpi t .
\end{aligned}
$$

For the calculation of the actual current density component $j_{r}$ according to formula (17), taking into account the Landau probability distribution, numerical methods were used. To this end, a computer program has been developed that calculates Landau distribution 


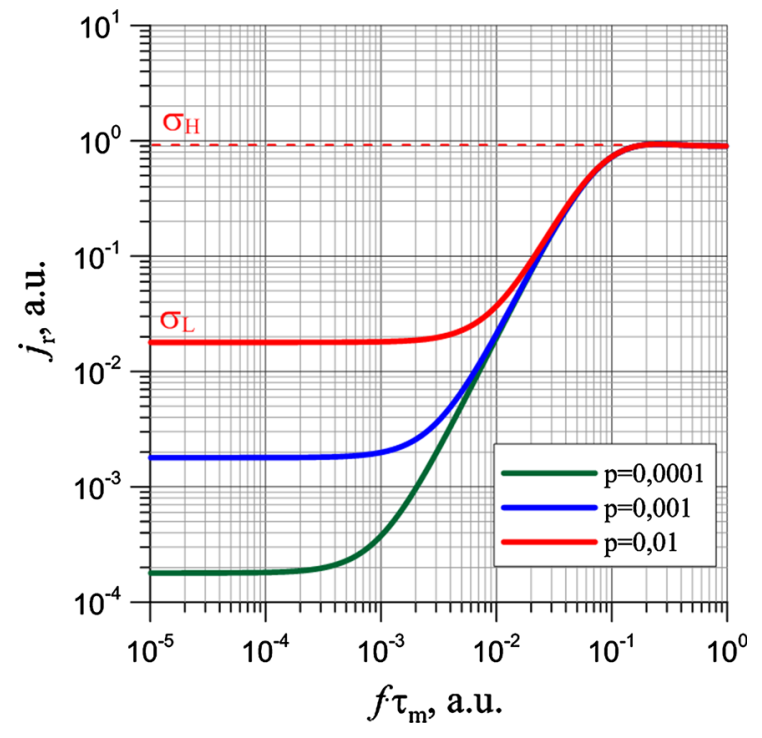

Fig. 4 The dependence of the actual current density component $j_{r}$ in the function of the product of the frequency values $f$ and the time $\tau_{m}$ for materials with a swift exchange of charges. Computer simulation for: (1) $p=0.01$; (2) $p=0.001$; (3) $p=0.0001$ (Źukowski et al. 2007)

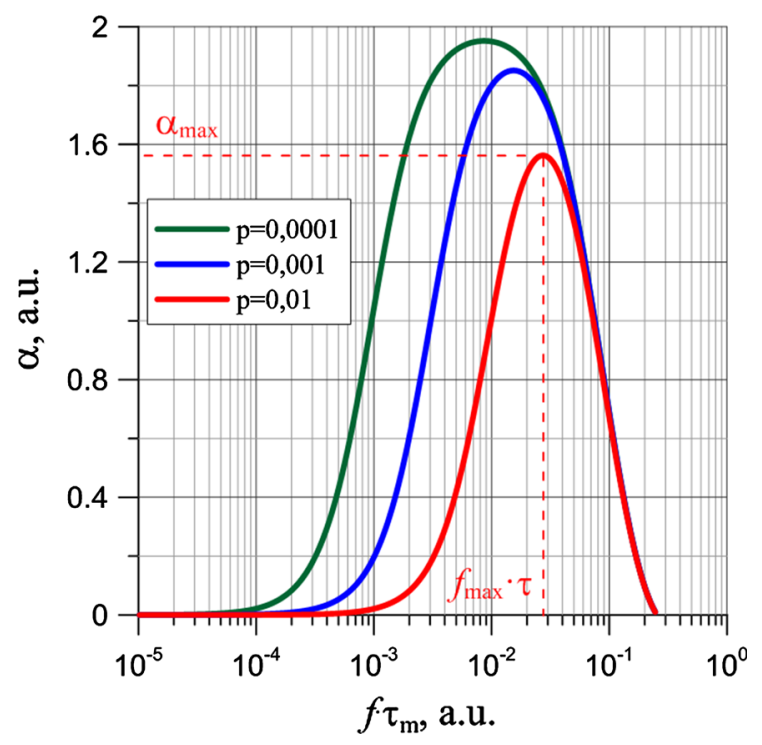

Fig. 5 The dependence of the frequency coefficient $\alpha$ versus the product of the values of frequency $f$ and time $\tau_{m}$ for materials with a swift exchange of charges. Computer simulation for: (1) $p=0.01$; (2) $p=0.001$; (3) $p=0.0001$ (Żukowski et al. 2007)

values for set values of expected hop time $\tau_{m}$ and standard deviation $\sigma_{m}$. Next, the program calculates the dependence of the real current density $j_{r}$ on the product of frequency $f$ and time $\tau_{m}$ for the Landau distribution for selected probability $\mathrm{p}$ values. The calculations were made for the probability value $p$ from $10^{-6}$ to 0.5 with a very small step. In Fig. 4, as an example, the calculated dependences $j_{r}(f)$ for the probability value $p=0.01 ; 0.001$ and 0.0001 are presented in the form of continuous lines. For the same values of probability $p$, the frequency coefficients $\alpha(f)$ were determined from formula (15) as a function of the product of frequency $f$ and time $\tau_{m}$. The exemplary dependences of the values of $\alpha(f)$ are shown in Fig. 5 as continuous lines.

As can be seen from Fig. 4, in the low frequency area $\sigma_{\mathrm{L}}(f)=$ const. The increase in frequency causes an increase in the conductivity, allowing for the fact that the slope of the growth section the value $\alpha_{\max }(f)$ in formula (15) decreases with the increase in the probability $p$ of a hop (Fig. 5), while in the high frequency region $\sigma_{\mathrm{H}}(f)=$ const.

As can be seen from Fig. 5, for small values of probability $p$ there are values of the frequency coefficient $\alpha_{\max }$ close to 2 . This corresponds to the situation when the hop takes place between wells with neutral charge. As a result of such a hop, the wells obtain opposite charges (an electric dipole is created), and the electric field of the dipole forces the electron to return to the first well. The low values of $\alpha$ correspond to the large probability of the p-jump. When the probability value rises to $p=0.5$ then the current density ceases to depend on the frequency of the alternating current and the value of $\alpha$ is equal to 0 .

The $p$ values included in formulas $(7-11,13,17)$ can be determined from the course $\sigma(f)$, shown, for example, in Fig. 5. From the analysis of formulas (13, 14) it follows that $p$ is the quotient of the conductivity values in the low $\sigma_{L}$ and high $\sigma_{H}$ regions frequency:

$p=\frac{\sigma_{L}}{2 \sigma_{H}}$.

The above analysis has shown that the key parameters of the hopping conductivity model at constant and alternating current are the probability p of hops and the lifetime of the dipole (relaxation time) $\tau$.

The experimental verification of the model, made for semiconductors containing potential wells and metallic phase nanoparticle composites in dielectric matrices [see, for example Kołtunowicz et al. (2013) and Svito et al. (2014)], showed both qualitative and quantitative compatibility of the measured results with 
the model of stepped transmission of electrons on a constant current and alternating.

The purpose of the work was to determine the formulas for the key parameters of the hopping conductivity model at direct and alternating current - the probability of leaks and relaxation time, to analyze the AC conductivity of the composite of cellulose, mineral oil and water nanoparticles based on the hopping conductivity model at direct and alternating current as well as to determine on the basis of $\mathrm{AC}$ conductivity measurements the dimensions of water nanodrops by using elements of quantum mechanics.

\section{The probability of hops and the lifetime of the dipole (relaxation time)}

The hopping probability $p$, entering formula (10) and the following ones, is one of the key parameters of the model of the swift exchange of charges at direct and alternating current. Below, the formula will be derived for its value, taking into account changes in potential energy during hops, shown in Figs. 1, 2 and 3.

As can be seen from Fig. 3, the probability of an electron hop after $\tau$ from the second to the first well in a weak electric field is:

$$
(1-p)=B \exp \left(\frac{\Delta W_{0}-U_{1}}{k T}\right)
$$

where $B$-numeric factor; $U_{1}$-value of the energy of the potential dipole resulting from the hop, given by the formula (Halliday and Resnick 1978)

$$
U_{1}=\frac{e}{4 \pi \varepsilon_{0} \varepsilon r_{1}},
$$

where $e$-the electron charge; $\varepsilon_{0}$-dielectric permittivity of the vacuum; $\varepsilon$-relative permittivity of the dielectric matrix, $r_{1}$-distance between neighbouring potential wells.

When the electron from the second well jumps to the third well, the probability $\mathrm{p}$ is (see Fig. 1):

$p=B\left(-\frac{U_{2}}{k T}\right)$,

where

$U_{2}=\frac{U_{1}}{2}$,

hence $p=\frac{p}{(1-p)+p}=\frac{\exp \left(-\frac{U_{1}}{2 k T}\right)}{\exp \left(\frac{\Delta W_{0}-U_{1}}{k T}\right)+\exp \left(-\frac{U_{1}}{2 k T}\right)}$.

Dividing the numerator and denominator of formula (23) by the value of $p$, given by formula (21), we obtain:

$p=\frac{1}{\exp \left(\frac{\Delta W_{0}}{k T}-\frac{U_{1}}{2 k T}\right)}$.

Formula (24) for weak fields, provided that $\Delta W_{0-}$ $-U_{1} / 2 \gg k T$, can be simplified to the form:

$p=\exp \left(-\frac{\Delta W_{0}}{k T}+\frac{U_{1}}{2 k T}\right)$.

Another important parameter of the model is the lifetime of the dipole $\tau$. As can be seen from Fig. 2, this time depends, in the first approximation, on the energy difference between the basic state (nanostructures with neutral charge) and the state of the negative charge. This is the same value that we call activation energy. Works (Psarras et al. 2003; Kudryashov et al. 2012) present the formula for the lifetime $\tau$ of the dipole:

$\tau=\tau_{0} \exp \left(\frac{\Delta W_{0}}{k T}\right)$

Figure 2 shows that the electron hop from the negative well to the positive one takes place with the emission of thermal energy with the value $\Delta W_{0}-U_{1}$ / 2. In the case of electron tunneling, the factor related to the speed of the fall of the wave function should also be taken into account. Hence the value of relaxation time is:

$$
\begin{aligned}
\tau & =\tau_{0} \exp \left(\beta \alpha r+\frac{\Delta W_{0}}{k T}-\frac{U_{1}}{2 k T}\right) \\
& =\tau_{0} \exp (\beta \alpha r) \exp \left(\frac{\Delta W}{k T}\right),
\end{aligned}
$$

where $\Delta W=\Delta W_{0}-U_{1} / 2$.

It follows from formula (27) that as the temperature rises, the frequency courses of conductivity, capacitance, loss tangent and frequency coefficient $\alpha(f)$ move parallel to each other into the area of higher frequencies of the forcing alternating current. This phenomenon can be taken into account by selecting the extremum on the experimental course and multiplying the measurement frequencies by the $\tau_{\max }$ value 
characteristic of the extreme. In this way Figs. 4 and 5 were made. This allows to recalculate the conductivity, capacity, loss angle tangent and frequency coefficient $\alpha$, obtained at any temperature, to the reference temperature of $20^{\circ} \mathrm{C}$ in electrotechnology and $0{ }^{\circ} \mathrm{C}$ in physics. The advantages of this method of converting experimental runs into the reference temperature were used in Żukowski et al. (2015b).

\section{The application of the model of direct and alternating current hopping conductivity for the analysis of the frequency dependencies of the conductivity of the composite of electrotechnical pressboard, mineral oil and water nanoparticles}

Figure 6 presents experimental the frequency dependencies of conductivity, dielectric permittivity and frequency coefficient $\alpha(f)$ determined for electrotechnical pressboard with a moisture content of $4 \%$ by weight, impregnated with insulating oil at a measuring temperature of $353 \mathrm{~K}$. On the course of conductivity, four frequency ranges can be distinguished, in which the changes in conductivity differ significantly. Namely, in the region of the lowest frequencies the

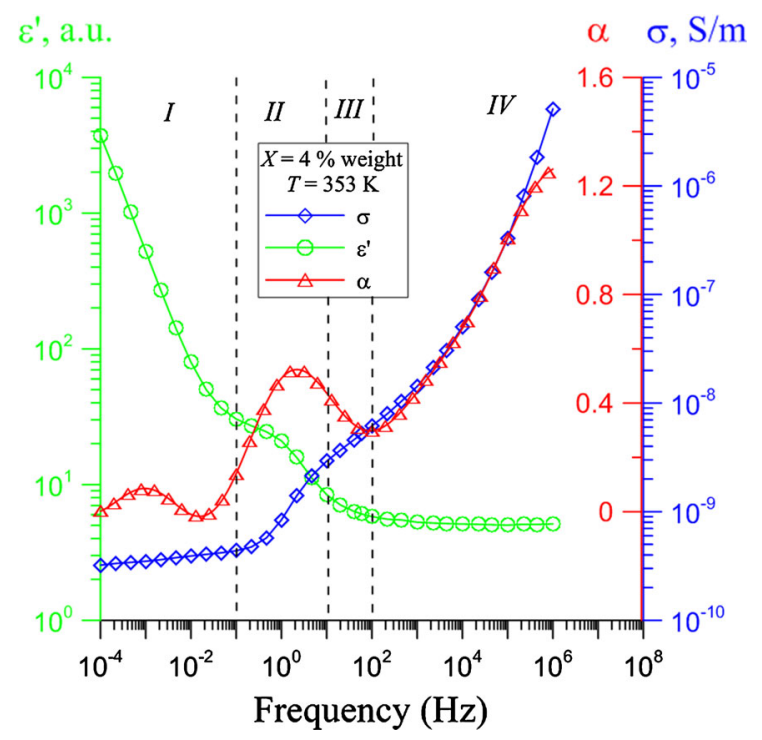

Fig. 6 Frequency dependencies of conductivity $\sigma(f)$ : (1) dielectric permeability, (2) frequency coefficient $\alpha(f)$, (3) for impregnated pressboard with water content $X=4 \%$ by weight. Measurement temperature $T=353 \mathrm{~K}$. Developed on the basis of (Żukowski et al. 2015b) conductivity is almost constant. After increasing the frequency to about $10^{-1} \mathrm{~Hz}$, the conductivity increases by almost one order. This increase is observed to the frequency of about $10 \mathrm{~Hz}$, and then it is slowing down. In the frequency range above $100 \mathrm{~Hz}$, another section of fast growth is observed. This means that in the composite of electrotechnical pressboard, mineral oil and water nanodrops there are at least two mechanisms of changes in conductivity. From the comparison experimental of the waveforms presented in Fig. 6 with the results of the computer simulation shown in Figs. 4 and 5, it can be seen that both the conductivity and the $\alpha(f)$ coefficient behave in the manner predicted by the hopping conductance model at direct and alternating current. On the course $\alpha(f)$ the maximum corresponding to the first section of the increase in conductivity is clearly visible. The maximal value $\alpha_{\max }$ for this growth section is $\alpha_{\max 1-}$ $\approx 0.5$. Based on formula (17), we can determine the value of the probability of $p_{1} \approx 0.2$. In the area up to about $1 \mathrm{~Hz}$ there are also increased dielectric permeability values, which means that in the area of lowest frequencies and the first section of growth there is electron tunneling between neutral water nanoparticles, which results in both increased conductivity and permittivity. As a result of such hops in the nanoparticle, there appears a molecule of water charged positively (the molecule from which the electron tunneled) or negatively (the one onto which it tunneled). The time of relaxation (time for the electron's return to the molecule from which it tunneled) can be estimated from the formula:

$\tau \approx \frac{1}{2 \pi f_{s}}$,

where $f_{\mathrm{s}}$ - the frequency at which the dependence $\sigma(f)$ reaches about half of the set value.

By substituting the frequency value $f_{\mathrm{s} 1}=1 \mathrm{~Hz}$, at which an increase in conductivity on the experimental course (Fig. 6) stops, we obtain $\tau_{1} \approx 0.02 \mathrm{~s}$.

The second segment of the increase in conductivity at frequencies above $100 \mathrm{~Hz}$ in the examined frequency range up to $10^{6} \mathrm{~Hz}$ did not reach steady state. This is also evident in the course of $\alpha(f)$, from which it follows that $\alpha_{\max 2}>1.2$. The probability of hops for the second growth section, calculated on the basis of formula (18), is $p_{2} \approx 0.0005$. As can be seen, it is about 400 times smaller than the probability of leaks for the first segment of growth. This means that the 
second section of the growth almost does not affect the DC conductivity or in the area of the lowest frequencies. Also, the relaxation time for the second growth is much shorter, and its value is $\tau_{2} \approx 2 \times 10^{-7} \mathrm{~s}$, that is, about $10^{5}$ times shorter than for the first stage of growth. The tunneling determining the second section of the conductivity increase does not increase the dielectric permittivity. It results from the formula for static dielectric permittivity caused by the hopping change of charges between neutral potential wells, derived in the work (Zukowski et al. 1997):

$\varepsilon=1+\chi=\frac{N P(T) \tau e^{2} r^{2}}{\varepsilon_{0} k T}$,

where $N$-concentration of potential wells; $P(T)$ - the probability of jumps in a unit of time; $\tau$-relaxation time; $r$-distance between potential wells; $e$-electron charge; $k$-Boltzmann constant; $T$-temperature.

It follows from formula (29) that with similar values of the potential well concentration at the first and second stage of conductivity, the dielectric permittivity should differ as many times as the relaxation time at the second stage is smaller than at the first stage, i.e. around $10^{5}$ times. This means that after the increase of the conductivity at the first stage and the transition to the second stage, the permeability of the composite electrotechnical pressboard, mineral oil and water nanoparticles should decrease to the value characteristic of the non-moisture composites. As can be seen from Fig. 6, after the end of the first stage of growth, there is indeed a decrease values determined experimentally in dielectric permittivity to about 5 .

As can be seen from Fig. 6, there are two stages of growth on the experimental frequency dependence of conductivity, low frequency and high frequency. These stages differ significantly in the values of relaxation times. The direct current conduction in the case of hopping conductivity takes place along the percolation channel that connects the measuring electrodes. In contrast, the conductivity of the alternating current can take place on much shorter sections and even, as in the case of the Debye model, in one polar molecule. For the case of two potential wells, when the frequency of alternating current is $1 / \tau$, the highest conductivity (current component density) will be observed. Further frequency increase almost does not change the value of AC conductivity (Fig. 4). The occurrence of two sections of the increase in conductivity is associated with the differences in distances between neighbouring nanoparticles in the composite of electrotechnical pressboard, mineral oil and water nanoparticles. For pressboard with a thickness of $1 \mathrm{~mm}$ the number of nanoparticles percolation channel connecting electrodes and the distances between neighboring nanoparticles them are in the order of $10^{5}$ and the average distance between them is around $10 \mathrm{~nm}$. According to the central limit theorem, for such a large number of randomly distributed elements, the probability distribution of the distance is approximately normal. This means that in the percolation channel there are pairs of neighbouring wells, the distances between which are both much smaller and much larger than the average. As can be seen from formula (27), in the case of a constant temperature, relaxation time depends in an exponential way on the distance the electron is tunneling through. As calculated above based on the experimental frequency dependence of conductivity, the relaxation time $\tau_{1}$ on the first (low-frequency) stretch of conductivity is about $10^{5}$-fold longer than on the high-frequency section $\left(\tau_{2}\right)$. Using the dependence on relaxation time (27) for the constant temperature $T=$ const. we can write:

$\frac{\tau_{1}}{\tau_{2}}=10^{5}=\exp \left[\frac{\beta}{R_{0}}\left(r_{1}-r_{2}\right)\right]$.

By substituting for formula (30) an experimentally determined value of the directional coefficientformula (5), it can be calculated that the distances between neighbouring potential wells that determine the conductivity on a high frequency conductivity rise are about $1.88 \mathrm{~nm}$ shorter than for the low frequency stage. Taking into account that the hop lengths have a probability distribution close to normal, and the average value is about $10 \mathrm{~nm}$ there is a high probability of the distance between potential wells differing by about $1.88 \mathrm{~nm}$.

Analysis of the experimental frequency dependencies of conductivity, permittivity and frequency coefficient $\alpha(f)$ for the composite electrotechnical pressboard-mineral oil-water nanoparticles showed that there is a high compatibility of these runs with the model of hopping conductivity at direct and alternating current of the composite of electrotechnical pressboard, mineral oil and water nanoparticles. This 
model is based on the quantum phenomenon of electron tunneling between potential wells. Using this fact, elements of quantum mechanics will be used below to determine the dimensions of water nanoparticles in electrotechnical pressboard impregnated with mineral insulating oil based on the analysis of the experimental dependences of conductivity on the water content.

\section{Determining the dimensions of nanodrops}

In work (Zukowski et al. 2014) based on laboratory tests it is clearly demonstrated that the direct current conductivity in moist impregnated isolation oil is determined by the moisture content and is carried out by electron tunneling between the nearest potential wells created by water molecules. This is due to the exponential dependence of the DC conductivity on the moisture content.

As shown in paper (Shklovskii and Efros 1984), in the case of electron tunneling between the closest potential wells there is a very strong (exponential) dependence of conductivity on the concentration of wells of potentials $N_{\mathrm{o}}$. In this case, dependence (5) can be used to analyze the dependence of conductivity on temperature and concentration of potential wells, provided that the activation energy does not depend on the concentration of the potential well. As was found in Żukowski et al. (2015b), the AC activation energy does not depend on the moisture content in the range of $1 \%$ by weight to $4 \%$ by weight and its value is $\Delta W \approx(1.0582 \pm 0.02224) \mathrm{eV}$. To apply formula (5) in the case of AC conductivity, one should select on the frequency dependencies for different moisture contents the frequency at which the conductivity depends only on the moisture content.

Figure 7 shows determined in laboratory tests the frequency dependencies of the conductivity of electrotechnical pressboard impregnated with insulating oil for the water content of $1 \%$ by weight, $2 \%$ by weight, $3 \%$ by weight, $4 \%$ by weight measured at 323 K. As shown in Fig. 7, in the region of the lowest frequencies steady state for a moisture concentration of $1 \%$ by weight has not yet been reached. For this concentration, the steady state can occur at frequencies lower than $10^{-4} \mathrm{~Hz}$ (outside the measurement range). This means that the waveforms shown in Fig. 7 are not satisfactory to determine the dependence of conductivity on the distance between water molecules according to formula (5). A similar situation occurs at the measuring temperature of $293 \mathrm{~K}$. Only for the measuring temperature of $353 \mathrm{~K}$, the courses for all moisture contents have a low-frequency section of the steady state (Fig. 8).

On the basis of the conductivity analysis, depending on the frequency, temperature and moisture content, the frequency and temperature appropriate for the application of formula (5) on the dependence of the hopping conductivity on the distance between potential wells are $10^{-4} \mathrm{~Hz}$ and $353 \mathrm{~K}$.

Similarly to work (Zukowski et al. 2014), we initially assume that potential wells are randomly distributed single molecules of water. The average distance $r_{1}$ between them is given by the formula:

$r_{1} \cong N_{0}^{-\frac{1}{3}}=\left(\frac{X_{\rho}}{100 u M_{\mathrm{H}_{2} \mathrm{O}}}\right)^{-\frac{1}{3}}$,

where $N_{0}$ - concentration of water molecules per unit volume; $\rho$-water weight density; $M_{\mathrm{H} 2 \mathrm{O}}=18$ molecular weight of water; $u=1.67 \times 10^{-27} \mathrm{~kg}-$ atomic mass unit; $X$-moisture, $\%$ by weight.

Observation of type (5) dependencies is a sufficient condition to determine the occurrence of electron

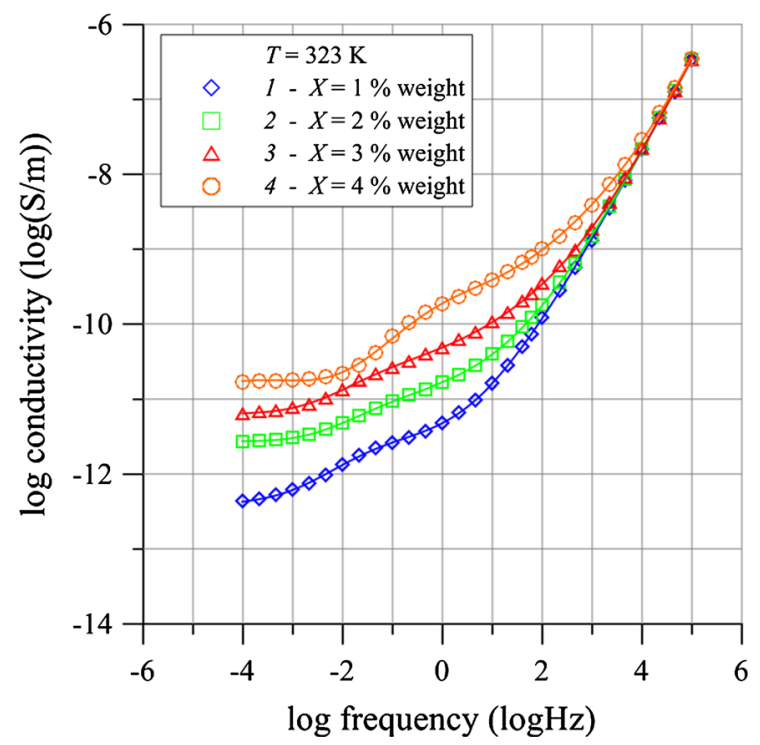

Fig. 7 Frequency dependencies of conductivity measured at a temperature of $323 \mathrm{~K}$ of electrotechnical pressboard impregnated with insulating oil for a water content of $1 \%$ by weight, $2 \%$ by weight, $3 \%$ by weight and $4 \%$ by weight 


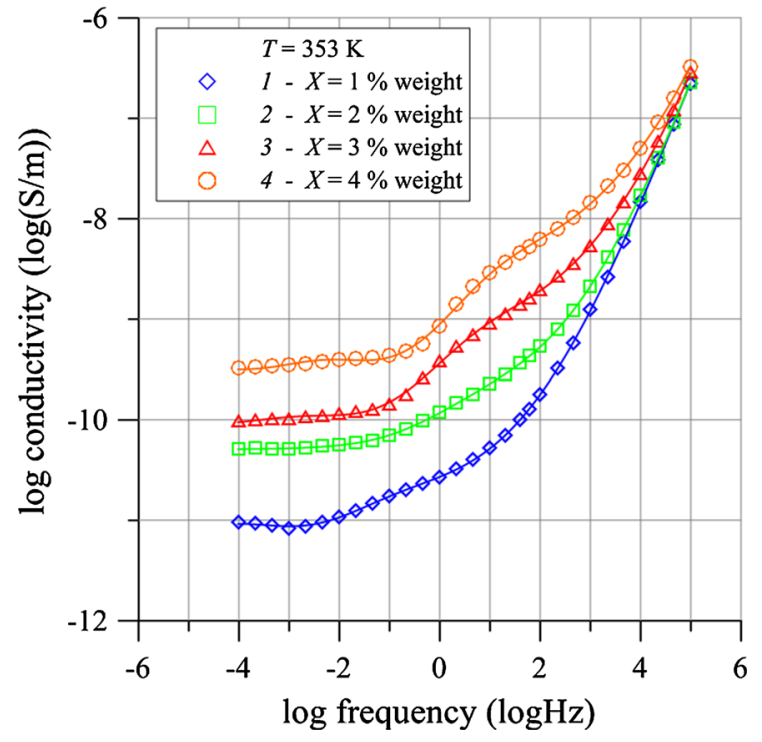

Fig. 8 Frequency dependencies of conductivity measured at a temperature of $353 \mathrm{~K}$ of electrotechnical pressboard impregnated with insulating oil for a water content of $1 \%$ by weight, $2 \%$ by weight, $3 \%$ by weight and $4 \%$ by weight

tunneling between the nearest potential wells (Shklovskii and Efros 1984). In the case of the independence of the activation energy from the moisture content (distances between water molecules), formula (5) can be transformed for the case of $T=$ const. into the following form:

$$
\begin{aligned}
\ln \left(r_{1}, T=\text { const } .\right) & =\left(\ln \sigma_{0}-\frac{\Delta W}{k T}\right)-\frac{\beta}{R_{0}} r_{1} \\
& =\frac{\beta}{R_{0}} r_{1}+C,
\end{aligned}
$$

where $C=\left(\ln \sigma_{0}-\frac{\Delta W}{k T}\right)$ and then experimental conductivity values can be plotted in the coordinates $\left\{\ln \sigma, r_{1}\right\}$. Figure 9 shows the dependence of the natural conductivity logarithm at $10^{-4} \mathrm{~Hz}$ and $353 \mathrm{~K}$ of the average distance between water molecules $r$. The approximation of this relationship with the least squares method.

As can be seen from Fig. 9, the relationship is a decreasing linear function, which is consistent with the formula for jump conductivity (5), a constant, within the uncertainty of measurement, directional coefficient $\beta / R_{0} \approx(-6.1667 \pm 0.23)$; the uncertainty of its determination is $\pm 3.63 \%$. The $R^{2}$ determination coefficient for the least squares approximation is close

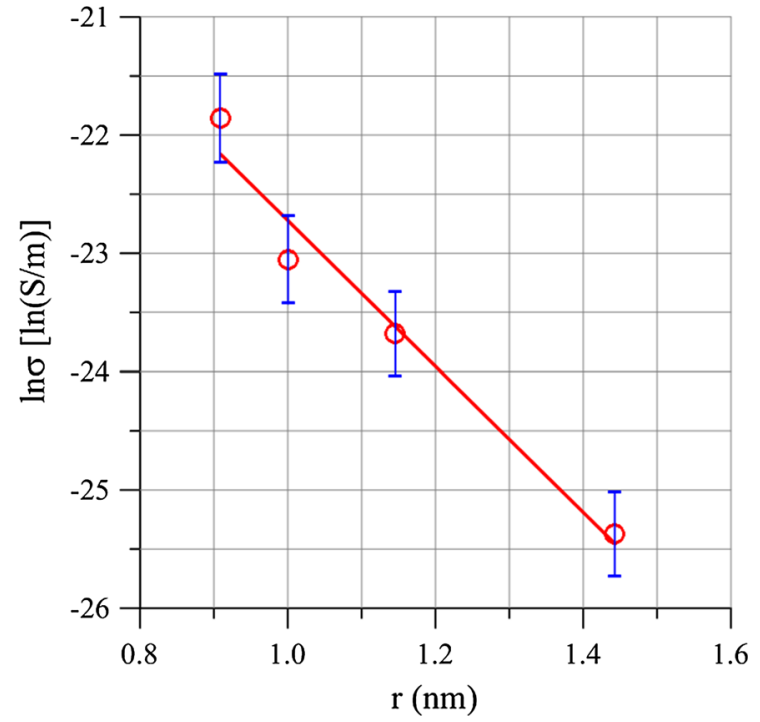

Fig. 9 Dependence of logarithm of natural conductivity at $10^{-4} \mathrm{~Hz}$ frequency and temperature of $353 \mathrm{~K}$ from the average distance between water molecules $r_{1}$ for the measurement temperature of $353 \mathrm{~K}$ and approximation by the least squares method

to one and equals $R^{2}=0.9672$, which proves the high accuracy of the approximation.

The approximation of the experimental course, shown in Fig. 9, allowed to obtain a formula for the approximating dependence:

$\ln \sigma=-6.1667 r_{1}-16.5556$

It is very important to determine the fact that the activation energy of direct current conductivity $\Delta W$ does not depend on the moisture content. Activation energy is a function of two energy states of the jumping electron-the initial state of the valence electron energy in the neutral well from which the electron begins its jump, and the first free state on which the electron will be in the second well after the jump (Fig. 1).

The radius of electron location for the water nanoparticle based on the Bohr model for the hydrogen atom will be calculated below. The formula (5) for conductivity in the case of electron tunneling will be used for calculations. The formula (5) includes the radius of the location of the hopping valence electron $R_{0}$ (so-called Bohr radius). For the calculation of the radius of the valence electron location of the water molecule, we will use an approach similar to the method of calculating the energy of ionization and the 
radius of the valence electron of an admixture of shallow phosphorus in semiconducting silicon described in Mott (1974).

In the case of the hydrogen atom, the only electron performs two functions the lowest (basic state) and the valence electron. Its ionization energy is $13.595 \mathrm{eV}$. It turns out that due to shielding in atoms containing several or more electrons, the valence electron is almost like in the field of a single proton, because for atoms with a nucleus load $Z$ between the valence electron and the nucleus there are $Z-1$ electrons. This causes the values of the ionization energy of the valence electron for the atoms of many elements and molecules to be similar to the energy of ionization of the hydrogen atom (Granovsky 1971; Finkelnburg and Humbach 1955; Watanabe 1957; Foner and Hudson 1956). Thus the ionization energies of neutral atoms of particular elements from the second period increase from $5.390 \mathrm{eV}$ for lithium to $24.580 \mathrm{eV}$ for the neon. The average value of ionization energy for atoms from the second period is about $13.5 \mathrm{eV}$. Similarly, in period 3, ionization energy rises from $5.198 \mathrm{eV}$ for sodium to $15.755 \mathrm{eV}$ for argon (Granovsky 1971). Here too, the average ionization energy has a similar value of around $13 \mathrm{eV}$. The ionization energy of many molecules is also similar to the ionization energy of the hydrogen atom. For example, for a water molecule it is equal to $12.590 \mathrm{eV}$, for oxygen molecules$12.075 \mathrm{eV}$, for carbon dioxide molecules$13.790 \mathrm{eV}$, for nitrogen molecules-15.600 eV (Finkelnburg and Humbach 1955; Watanabe 1957; Foner and Hudson 1956; Price 1947). This means that a valence electron in an atom of an element or a molecule can in many cases be, with some approximation, treated in a similar way as an electron in a hydrogen atom. This can be explained by the fact that the valence electron is effectively shielded from the nucleus by the underlying electrons, so that its energy is similar to the energy of the electron in the field of a single proton. In addition, the value of energy is influenced by the distance of the valence electron, which slightly changes with the change of the atomic number $Z$. The conclusion about the similarity of the valence electrons of elements with $Z>1$ to the state of electron in a hydrogen atom is very important and used for many decades in theory semiconductors for calculating the ionization energy of shallow admixtures (Mott 1974). It turns out that the energy states of the atoms of the donor impurity phosphorus in silicon can be described with a relatively good approximation using the formula for the hydrogen state of the hydrogen atom in the Bohr model (Mott 1974). The model used for this purpose is called the hydrogen-like model of shallow-lying impurities. In this model, it was considered, first, that the valence electron of the impurity is screened by lower electrons, so that it is in the field of a single proton. Second, the phosphorus atom is placed in silicon with relative dielectric permittivity $\varepsilon$, whose value is 12.5 (Kiriejew 1971). Considering this, in the formula for the energies of the states of the electron in a hydrogen atom, the so-called Bohr model, one should place in the nominative $\varepsilon_{0}^{2}$ next to $\varepsilon^{2}$ (Halliday and Resnick 1978):

$E_{n}=\frac{e^{4} m_{e}}{8 \varepsilon_{0}^{2} \varepsilon_{r}^{2} h^{2} n^{2}}$,

where $n=1,2,3 \ldots$ is an integer called the main quantum number; $m_{\mathrm{e}}$-mass of electron; $e$-electron charge; $\varepsilon_{0}$-dielectric permittivity of the vacuum; $\varepsilon_{\mathrm{r}}$ dielectric permittivity of silicon; $h$-Planck constant.

Next, it is assumed that the valence electron is at the level of $n=1$. The first ionization energy of the phosphorus atom is $E_{1}=10.49 \mathrm{eV}$ (Buhl 1994). According to formula (34), the ionization energy of the phosphorus atom in the silicon atom should decrease $\varepsilon^{2}=156.25$ times to the level of $0.067 \mathrm{eV}$. Comparing this result with the experienced ionization energy value of the phosphorus atom in $0.045 \mathrm{eV}$ silicon (Buhl 1994), it can be concluded that the difference between experimental and calculated values is less than 1.5 times. This is a satisfactory outcome. As a result, shallow admixtures, such as phosphorus in silicon, are often called hydrogen-like admixtures. The difference between the experimental and theoretical values is probably connected with the fact that near the atom of the admixture of phosphorus, the polarization of silicon differs slightly from the state found in the ideal network that does not contain admixtures and defects. From formula (34) it follows that to obtain ionization energy $0.045 \mathrm{eV}$, the dielectric permeability close to the phosphorus atom should be about 10.5. This is a reduction of only $16 \%$ compared to pure silicon.

In the case of the phosphorus atom in the silicon, ionization energy was calculated. However, we know the energy of activation of conductivity, on the basis of which we can calculate the dielectric permittivity of 
the material, surrounded by which the water nanoparticle is located, from which the electron is tunneling. In the case of tunneling, the energy of activation is, in a good approximation, the energy between the fundamental state of the valence electron of the water molecule and the state to which the electron hops, transforming the neutral molecule of water into a negatively charged one, i.e. the first unfilled state. This is due to Pauli's principle, because the state from which the electron from the neutral first nanoparticle tunnels is occupied by the electron in the neutral other nanoparticle. Therefore, the electron after the hop will be on the first lowest vacancy in the second nanoparticle. This situation is shown in Fig. 10.

We shall build on the following assumptions.

The electron is tunneling from an inert molecule of water that is in a nanodrop. Tunneling takes place from the ground state to the first higher unoccupied state.

Due to the fact that the valence electron of a molecule of water is strongly shielded from the nucleus through lower electrons, its first ionisation energy is about 12.6 eV (Mallard and Linstrom 2000; Shirai et al. 2001; Zavilopulo et al. 2005; Kovtun 2015) and is very similar to the ionization energy of a hydrogen atom of 13,595 eV. The difference between these values is only $7.3 \%$. Such close values of the ionization energy of the water molecule and the hydrogen atom mean that the structure of the energy states of the water molecule's valence electron is similar to the structure of the hydrogen atom states.

In order to calculate the energy difference between the basic state and the first unfulfilled state of the water molecule, we shall use the Bohr formula for energy between states in the hydrogen atom (Halliday and Resnick 1978):

$h v_{j, k}=E_{j}-E_{k}=\frac{e^{4} m_{e}}{8 \varepsilon_{0}^{2} \varepsilon_{r}^{2} h^{2}}\left(\frac{1}{j^{2}}-\frac{1}{k^{2}}\right)$,

where $m_{e}$-the mass of the electron; $e$-electron charge; $\varepsilon_{0}$-dielectric permittivity of the vacuum; $\varepsilon_{\mathrm{r}}$ relative dielectric permittivity of the material; $h-$ Planck constant; $j$ - the main quantum number of the state of the valence electron; $k$ - the main quantum number of the lowest unoccupied state.

Dielectric permittivity of pressboard impregnated with insulating oil $\varepsilon_{\mathrm{r}} \neq 1$. Thus, in formula (35) in the denominator there is a square of relative permittivity.

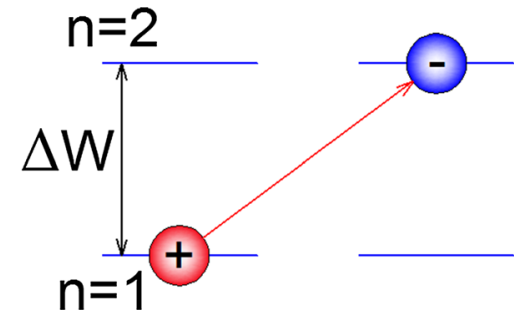

Fig. 10 Basic state $(n=1)$ and the first unoccupied state $(n=2)$ between which an electron hops from one neutral potential well to another

In formula (35), similarly as in the model of the hydrogen-like admixture of the phosphorus atom in silicon, we assume that the valence electron of the water molecule is in the ground state with the main quantum number $j=1$. In contrast to the model of the hydrogen-like admixture, where the ionization energy is calculated, i.e. the electron's hop into the state of $k$ equal to infinity, in formula (35) in the case of tunneling for the first unoccupied state, one should write $k=2$.

By substituting for formula (35) the values of $j=1$ and $k=2$ and the DC activation energy known to us, $\Delta W=h v$, we can calculate the relative dielectric permittivity in the environment of the water molecule to which the electron tunnels:

$\varepsilon_{r}=\frac{e^{2}}{h \varepsilon_{0}} \sqrt{\frac{3 m_{e}}{32 \Delta W}}$.

Substituting for formula (36) the value of AC activation energy, which is $\Delta W \approx(1.05820 \pm$ 0.2224) eV (Żukowski et al. 2015b), and known fixed values: $m_{e}, e, \varepsilon_{0}, h$, we obtain the relative permittivity of the insulating material around the water molecule, which the electron is tunneling. Its value is $\varepsilon_{r} \approx 5.62$. This is a value similar to the dielectric permittivity of the pressboard in the frequency range above $1000 \mathrm{~Hz}$. The obtained value of the relative permittivity of the material surrounding the molecule of water on which the electron is tunneling indicates that the molecule of water from which the electron tunnels is not in the insulating oil filling the spaces between the cellulose fibers. The dielectric permittivity value of the relative insulating oil is much lower and amounts to approximately 2.2 (CIGRE Working Group A2.35 2010). This means that the moisture nanoparticles are located inside the cellulose fibers, 
the dielectric permittivity of which is greater than the dielectric permittivity of the oil.

The radius of Bohr's first orbit $(n=1)$ for a hydrogen atom in a vacuum is $0.0529 \mathrm{~nm}$ (Halliday and Resnick 1978): and is given by the formula:

$R_{n}=\frac{\varepsilon_{0}^{2} \varepsilon_{r}^{2} h^{2} n^{2}}{\pi e^{2} m_{e}}$,

where $\varepsilon_{\mathrm{r}}=1$.

When a molecule of water from which an electron hops, is placed in pressboard impregnated with insulation oil with a relative permittivity $\varepsilon_{\mathrm{r}} \approx 5.62$, the Bohr radius, entering the formula for hopping conductivity (5) will increase $\left(\varepsilon_{\mathrm{r}}\right)^{2}$ times, and its value will be $R_{0} \approx 1.657 \mathrm{~nm}$.

The experimentally determined value of the direction coefficient $\beta / R_{0}$, entering formula (5) for conductivity in the case of electron tunneling, calculated on the assumption that the moisture in the pressboard is in the form of individual water molecules, is 6.1667 and is described by the formula:

$\left(\frac{\beta}{R_{0}}\right)_{\exp } r_{1}=6.1667 r_{1}$,

where $\beta \approx(1.75 \pm 0.05)$ (Shklovskii and Efros 1984), $r_{1}$ - distance calculated on the assumption that the moisture in the pressboard is in the form of individual water molecules-formula (31).

Taking into account the fact that the water in the pressboard is in the form of nanodrops, the distance to which the electron tunnels is different than $r_{1}$. We will mark it $r_{\mathrm{n}}$.

The average distance between single molecules of water $r$ is determined by the moisture content using formula (31). When nanodrops are found to contain $n$ nanomolecules, the average distance between nanodrops is $r_{\mathrm{n}}$ and is determined by the formula:

$r_{n} \cong\left(\frac{X_{\rho}}{100 u M_{\mathrm{H}_{2} \mathrm{O}}}\right)^{-\frac{1}{3}}=r_{1} \sqrt[3]{n}$,

where $n$-the average number of water molecules in the nanodroplet; $\rho$-mass density; $M_{\mathrm{H} 2 \mathrm{O}}=18$ molecular weight of water; $u=1.67 \times 10^{-27} \mathrm{~kg}-$ atomic mass unit; $X$-moisture, $\%$ by weight.

By substituting in formula (31) the value $R_{0} \approx 1.657 \mathrm{~nm}$, calculated above, we should simultaneously increase the distance at which the electron will hop to the value of $r_{n}$. By substituting in formula
(31) the values of $\beta$ and $R_{0} \approx 1.657 \mathrm{~nm}$, calculated above from the Bohr model, we obtain:

$\left(\frac{\beta}{R_{0}}\right)_{\text {Bohr }} r_{n}=1.056 r_{n}$.

Comparing the values of the directional coefficient, being part of formula (31): the experimental one (31) and the one calculated from the Bohr model (40), we conclude that the distance between the molecule from which the electron hops and the molecule it hops onto should be much greater than if the moisture was in the pressboard in the form of individual molecules. This means that the water molecules are in the pressboard in the form of nanodrops, each of which contains an average of $n$ water molecules. The average distances between water nanoparticles will increase in this case

$\frac{r_{n}}{r_{1}} \cong\left(\frac{\beta}{R_{0}}\right)_{\exp } /\left(\frac{\beta}{R_{0}}\right)_{\text {Bohr }}=5.839$.

Assuming that the water molecules accumulate in the pressboard impregnated with insulating oil in the form of nanodrops, and each nanodrop contains an average of $n$ molecules of water, formula (41) for distance $r_{n}$ between nanoparticles should be transformed into the form:

$\frac{r_{n}}{r_{1}} \cong(n)^{1 / 3}$

From formula (42) it follows that:

$n=\left(\frac{r_{n}}{r_{1}}\right)^{3}$.

By substituting in formula (43) formula (41), we obtain water nanoparticles that contain on average about 200 molecules of water. The diameter of such nanodrops is about $2.24 \mathrm{~nm}$. When comparing the number of water molecules in nanodrops, determined on the basis of semi-empirical calculations with the $\mathrm{X}$-ray of a double-negative oxygen ion, amounting to about 220 and their diameters $2.32 \mathrm{~nm}$ (Źukowski et al. 2015a) with that obtained by elements of quantum mechanics (about 200), it can be seen that these last calculations give the number of water molecules in nanodrops about 0.91 times lower, their diameter being about $2.24 \mathrm{~nm}$. This is consistent with the sufficient condition for the formation of nanoparticles (Pogrebnjak and Beresnev 2012; Pogrebnjak et al. 2018), which is that oil and water do not enter 
chemical reactions, they are not mutually soluble and the surface tension of water is much higher than that of insulating oil.

It seems that the calculation of nanodrop, made on the basis of laboratory tests low-frequency conductivity with made using the basics of quantum mechanics are more reliable, because they allow to determine both the dimensions of the nanodrops and calculate the dielectric permittivity of the pressboard impregnated with insulating oil.

Semi-empirical calculations and the use of elements of quantum mechanics made on the basis of laboratory tests of DC and AC conductivity of pressboard impregnated with insulating oil, clearly demonstrate that the moisture in pressboard is in the form of nanodrops. Therefore, such material should be treated as a composite of cellulose, mineral oil and water nanoparticles.

\section{Conclusion}

The paper presents a model of hopping conductivity at direct and alternating current, developed on the basis of the quantum phenomenon of electron tunneling between neighbouring potential wells. The tunneling takes place from one neutral potential well to another, resulting in an electrical dipole and additional thermally activated polarization. An important parameter of the model is the dipole lifetime $\tau$. After this time, the electron can hop to the third well with the probability $p$, which causes a direct current flow, or return to the first well with the probability $(1-p)$, thus causing the flow of high-frequency current. The model shows that for direct or low frequency current, the current density and conductivity do not depend on the frequency. In the high frequency region, the current density does not depend on the frequency. Low-frequency conductivity is $2 p$ times smaller than high-frequency conductivity. In the transitional region there is a frequency dependence of the current density.

An analysis designated in laboratory tests of the frequency dependence of conductivity and permittivity for the composite of electrotechnical pressboard, mineral oil and water nanoparticles was made with the use of the direct and alternating current hopping conductivity model, which showed that there is a high correspondence between the waveforms and the model. It has been established that in the composite of electrotechnical pressboard, mineral oil and water nanocrusts there are at least two mechanisms of changes in conductivity. For the first stage, the value of the hopping probability $p_{1} \approx 0.2$, and the value of the relaxation time $\tau_{1} \approx 0.02 \mathrm{~s}$. The probability of hops for the second stage of growth is $p_{2} \approx 0.0005$ and the value of relaxation time $\tau_{2} \approx 2 \times 10^{-7} \mathrm{~s}$. The occurrence of two sections of the conductivity increase is associated with differences in distances between neighbouring nanoparticles obtaining in the composite of electrotechnical pressboard, mineral oil and water nanodrops. In the percolation channel, connecting measuring electrodes for pressboard with a thickness of $1 \mathrm{~mm}$, the number of nanoparticles and number the distance between the neighboring molecules them is in the order of $10^{5}$. According to the central limit theorem, for such a large number of randomly distributed elements, the probability distribution of the occurrence of distance is approximately normal. This means that in the percolation channel there are pairs of neighbouring wells, the distances between which are both much smaller and much larger than the average. A pair of wells, the distance between which is smaller than average, participates in the permittivity with a short relaxation time. On the other hand, a pair of neighbouring wells for which the distance is greater than average causes a high resistance to DC or low-frequency current as well as a higher relaxation time.

Using the elements of quantum mechanics on the basis in the laboratory tests of the dependences of lowfrequency conductivity from the moisture content was determined, the dimensions of water nanoparticles in electrotechnical pressboard impregnated with mineral insulating oil were determined. It was found that nanoparticles contain on average about 200 molecules of water, and their diameters are about $2.24 \mathrm{~nm}$. This approach allowed to calculate the dielectric permittivity of cellulose, the value of which is $\varepsilon_{\mathrm{r}} \approx 5.62$. It is a value close to the static dielectric permittivity of the pressboard and is much higher than the permittivity of the insulating oil for which $\varepsilon_{\mathrm{r}} \approx 2.2$. The obtained value of the relative permittivity of the material surrounding the molecule of water to which the electron tunnels indicates that the water nanoparticles between which the electron is tunneling are inside the cellulose fibers and not in the insulating oil that fills the spaces between the cellulose fibers. 
Open Access This article is distributed under the terms of the Creative Commons Attribution 4.0 International License (http:// creativecommons.org/licenses/by/4.0/), which permits unrestricted use, distribution, and reproduction in any medium, provided you give appropriate credit to the original author(s) and the source, provide a link to the Creative Commons license, and indicate if changes were made.

Funding The funding was provided by Polish Ministry of Science and Higher Education [Grand No: Statute tasks-8620/E361/S/2018 (S-28/E/2018)].

\section{References}

Buhl F (1994) Poradnik chemika analityka. T. 1, Dane fizykochemiczne, analiza chemiczna. Wydawnictwa NaukowoTechniczne

CIGRE Working Group A2.35 (2010) Experiences in service with new insulating liquids, CIGRE Brochure No 436

Cole KS, Cole RH (1941) Dispersion and absorption in dielectrics I. Alternating current characteristics. J Chem Phys 9:341-351. https://doi.org/10.1063/1.1750906

Cole KS, Cole RH (1942) Dispersion and absorption in dielectrics II. Direct current characteristics. J Chem Phys 10:98-105. https://doi.org/10.1063/1.1723677

Dey D, Chatterjee B, Chakravorti S, Munshi S (2010) Importance of denoising in dielectric response measurements of transformer insulation: an uncertainty analysis based approach. Measurement 43:54-66. https://doi.org/10.1016/ J.MEASUREMENT.2009.06.009

Ekanayake C, Gubanski SM, Graczkowski A, Walczak K (2006) Frequency response of oil impregnated pressboard and paper samples for estimating moisture in transformer insulation. IEEE Trans Power Deliv 21:1309-1317. https:// doi.org/10.1109/TPWRD.2006.877632

Finkelnburg W, Humbach W (1955) Ionisierungsenergien von atomen und atomionen. Naturwissenschaften 42:35-37

Foner SN, Hudson RL (1956) Ionization potential of the OH free radical by mass spectrometry. J Chem Phys 25:602-603. https://doi.org/10.1063/1.1743001

Gilbert R, Jalbert J, Duchesne S et al (2010) Kinetics of the production of chain-end groups and methanol from the depolymerization of cellulose during the ageing of paper/ oil systems. Part 2: thermally-upgraded insulating papers. Cellulose 17:253-269. https://doi.org/10.1007/s10570009-9365-2

Granovsky V (1971) Electric current in gas. Steady-state current. Nauka 70:83

Halliday D, Resnick R (1978) Physics part II. Wiley, New York

Havriliak S, Havriliak SJ (1997) Dielectric and mechanical relaxation in materials: analysis, interpretation, and application to polymers. Hanser Publishers, Munich

Jalbert J, Rodriguez-Celis E, Duchesne S et al (2015) Kinetics of the production of chain-end groups and methanol from the depolymerization of cellulose during the ageing of paper/ oil systems. Part 3: extension of the study under temperature conditions over $120{ }^{\circ} \mathrm{C}$. Cellulose 22:829-848. https:// doi.org/10.1007/s10570-014-0516-8
Jaya M, Geissler D, Leibfried T (2013) Accelerating dielectric response measurements on power transformers-part I: a frequency-domain approach. IEEE Trans Power Deliv 28:1469-1473. https://doi.org/10.1109/TPWRD.2013.225 3135

Jonscher AK, Andrzej K (1983) Dielectric relaxation in solids. Chelsea Dielectrics Press, London

Kiriejew PS (1971) Fizyka półprzewodników. Polskie Wydawnictwo Naukowe, Warszawa

Kołtunowicz TN, Fedotova JA, Zhukowski P et al (2013) Negative capacitance in (FeCoZr)-(PZT) nanocomposite films. J Phys D Appl Phys 46:125304. https://doi.org/10. 1088/0022-3727/46/12/125304

Kovtun YV (2015) Mean energy of water molecule ionization by electron impact. Tech Phys 60:1110-1118. https://doi. org/10.1134/S1063784215080162

Kudryashov MA, Mashin AI, Logunov AA et al (2012) Frequency dependence of the electrical conductivity in $\mathrm{Ag} /$ PAN nanocomposites. Tech Phys 57:965-970. https://doi. org/10.1134/S1063784212070134

Mallard WG, Linstrom PJ (2000) NIST standard reference database E, vol.69. http://webbook.nist.gov

Mott NF (1974) Metal-insulator transitions. Taylor and Francis, London

Mott NF, Gurney RW (1950) Electronic processes in ionic crystals. At the Clarendon Press, Oxford

Mott NF, Nevill F, Davis EA, Edward A (1979) Electronic processes in non-crystalline materials, 2nd edn. Clarendon Press, New York

Nowak R (2002) Statystyka dla fizyków. Wydaw. Naukowe PWN

Oommen TV (1983) Moisture equilibrium in paper oil systems. In: Proceedings of the 16th electrical/electronics insulation conference

Pogrebnjak AD, Beresnev VM (2012) Nanocoatings, nanosystems, nanotechnologies. Bentham Science, Oak Park

Pogrebnjak AD, Ivashchenko VI, Skrynskyy PL et al (2018) Experimental and theoretical studies of the physicochemical and mechanical properties of multi-layered $\mathrm{TiN} / \mathrm{SiC}$ films: temperature effects on the nanocomposite structure. Compos Part B Eng 142:85-94. https://doi.org/10.1016/J. COMPOSITESB.2018.01.004

Price WC (1947) The effect of alkyl substitution on the spectra and ionization potentials of some fundamental chromophores. Chem Rev 41:257-272

Psarras GC, Manolakaki E, Tsangaris GM (2003) Dielectric dispersion and ac conductivity in-Iron particles loadedpolymer composites. Compos Part A Appl Sci Manuf 34:1187-1198. https://doi.org/10.1016/J.COMPOSIT ESA.2003.08.002

Ravich YI, Nemov SA (2002) Hopping conduction via strongly localized impurity states of indium in $\mathrm{PbTe}$ and its solid solutions. Semiconductors 36:1-20. https://doi.org/10. $1134 / 1.1434506$

Saha TK (2003) Review of time-domain polarization measurements for assessing insulation condition in aged transformers. IEEE Trans Power Deliv 18:1293-1301. https:// doi.org/10.1109/TPWRD.2003.817741

Saha TK, Purkait P (2004) Investigation of polarization and depolarization current measurements for the assessment of oil-paper insulation of aged transformers. IEEE Trans 
Dielectr Electr Insul 11:144-154. https://doi.org/10.1109/ TDEI.2004.1266329

Shirai T, Tabata T, Tawara H (2001) Analytic cross sections for electron collisions with $\mathrm{Co}, \mathrm{Co}_{2}$, And $\mathrm{H}_{2} \mathrm{O}$ relevant to edge plasma impurities. At Data Nucl Data Tables 79:143-184. https://doi.org/10.1006/ADND.2001.0866

Shklovskii BI, Efros AL (1984) Electronic properties of doped semiconductors. Springer, Berlin

Svito I, Fedotov AK, Koltunowicz TN et al (2014) Hopping of electron transport in granular $\mathrm{Cu}_{x}\left(\mathrm{SiO}_{2}\right)_{1-x}$ nanocomposite films deposited by ion-beam sputtering. J Alloys Compd 615:S371-S374. https://doi.org/10.1016/J.JALLCOM. 2014.01.136

Walczak K, Graczkowski A, Gielniak J et al (2006) Dielectric frequency response of cellulose samples with various degree of moisture content and aging. Prz Elektrotechn $1: 264-267$

Watanabe K (1957) Ionization potentials of some molecules. J Chem Phys 26:542-547. https://doi.org/10.1063/1.174 3340

Wolny S, Kędzia J (2010) The assessment of the influence of temperature of selected parameters of the approximation method of depolarization current analysis of paper-oil insulation. J Non Cryst Solids 356:809-814. https://doi. org/10.1016/J.JNONCRYSOL.2009.07.046

Zavilopulo AN, Chipev FF, Shpenik OB (2005) Ionization of nitrogen, oxygen, water, and carbon dioxide molecules by near-threshold electron impact. Tech Phys 50:402. https:// doi.org/10.1134/1.1901776

Zhang T, Li X, Lv H, Tan X (2014) Parameter identification and calculation of return voltage curve based on FDS data.
IEEE Trans Appl Supercond 24:1-5. https://doi.org/10. 1109/TASC.2014.2344763

Zukowski PW, Rodzik A, Shostak Y (1997) Dielectric constant and ac conductivity of semi-insulating $\mathrm{Cd}_{[1-\mathrm{x}]} \mathrm{Mn}_{[x]} \mathrm{Te}$ semiconductors. Semiconductors 31:610-614. https://doi. org/10.1134/1.1187227

Zukowski P, Koltunowicz TN, Kierczynski K et al (2014) Assessment of water content in an impregnated pressboard based on DC conductivity measurements theoretical assumptions. IEEE Trans Dielectr Electr Insul 21:12681275. https://doi.org/10.1109/TDEI.2014.6832274

Żukowski P, Kołtunowicz T, Partyka J et al (2007) Dielectric properties and model of hopping conductivity of GaAs irradiated by $\mathrm{H}+$ ions. Vacuum 81:1137-1140. https:// doi.org/10.1016/J.VACUUM.2007.01.070

Żukowski P, Kołtunowicz TN, Kierczyński K et al (2015a) Formation of water nanodrops in cellulose impregnated with insulating oil. Cellulose 22:861-866. https://doi.org/ 10.1007/s10570-015-0543-0

Żukowski P, Kołtunowicz TN, Kierczyński K et al (2015b) An analysis of AC conductivity in moist oil-impregnated insulation pressboard. IEEE Trans Dielectr Electr Insul 22:2156-2164. https://doi.org/10.1109/TDEI.2015. 004606

Publisher's Note Springer Nature remains neutral with regard to jurisdictional claims in published maps and institutional affiliations. 